Bull. Korean Math. Soc. 48 (2011), No. 6, pp. 1315-1327

http://dx.doi.org/10.4134/BKMS.2011.48.6.1315

\title{
JACOBI OPERATORS ALONG \\ THE STRUCTURE FLOW ON REAL HYPERSURFACES IN A NONFLAT COMPLEX SPACE FORM II
}

\author{
U-HANG Ki AND Hiroyuki KuriHara
}

\begin{abstract}
Let $M$ be a real hypersurface of a complex space form with almost contact metric structure $(\phi, \xi, \eta, g)$. In this paper, we study real hypersurfaces in a complex space form whose structure Jacobi operator $R_{\xi}=R(\cdot, \xi) \xi$ is $\xi$-parallel. In particular, we prove that the condition $\nabla_{\xi} R_{\xi}=0$ characterizes the homogeneous real hypersurfaces of type $A$ in a complex projective space or a complex hyperbolic space when $R_{\xi} \phi S=$ $R_{\xi} S \phi$ holds on $M$, where $S$ denotes the Ricci tensor of type $(1,1)$ on $M$.
\end{abstract}

\section{Introduction}

Let $\left(M_{n}(c), J, \tilde{g}\right)$ be a complex $n$-dimensional complex space form with Kähler structure $(J, \tilde{g})$ of constant holomorphic sectional curvature $4 c$ and let $M$ be an orientable real hypersurface in $M_{n}(c)$. Then $M$ has an almost contact metric structure $(\phi, \xi, \eta, g)$ induced from $(J, \tilde{g})$.

Typical examples of real hypersurfaces in $M_{n}(c), c \neq 0$, are homogeneous ones. Takagi [12], [13] classified homogeneous real hypersurfaces of a complex projective space $P_{n} \mathbb{C}$ into six model spaces. On the other hand, Cecil and Ryan [2] extensively studied a Hopf hypersurface, which is realized as tubes over certain submanifolds in $P_{n} \mathbb{C}$, by using its focal map. By making use of those results and the mentioned work of Takagi, Kimura [7] proved the local classification theorem for Hopf hypersurfaces of $P_{n} \mathbb{C}$ all of whose principal curvatures are constants. For the case where a complex hyperbolic space $H_{n} \mathbb{C}$, Berndt [1] proved the classification theorem for Hopf hypersurfaces whose all principal curvatures are constants. Among the several types of real hypersurfaces appeared in model spaces ([1], [12]), a particular type of tubes over totally geodesic $P_{k} \mathbb{C}$ or $H_{k} \mathbb{C}(0 \leq k \leq n-1)$ adding a horosphere in $H_{n} \mathbb{C}$, which is called type $A$, has a lot of nice geometric properties. For example, Okumura [9] (resp. Montiel and Romero [8]) showed that a real hypersurface in $P_{n} \mathbb{C}$ (resp.

Received August 17, 2010; Revised June 1, 2011.

2010 Mathematics Subject Classification. Primary 53B20, 53C15, 53C25.

Key words and phrases. complex space form, real hypersurface, structure Jacobi operator, Ricci tensor. 
$H_{n} \mathbb{C}$ ) is locally congruent to one of real hypersurfaces of type $A$ if and only if the Reeb flow $\xi$ is isometric or equivalently the structure operator $\phi$ commutes with the shape operator $H$.

The structure Jacobi operator $R_{\xi}=R(\cdot, \xi) \xi$ has a fundamental role in contact geometry. Ortega, Pérez and Santos [10] have proved that there are no real hypersurfaces in $P_{n} \mathbb{C}, n \geq 3$ with parallel structure Jacobi operator $\nabla R_{\xi}=0$. More generally, such a result has been extended by [11] due to them. In this paper, motivated by results mentioned above we consider the parallelism of the structure Jacobi operator $R_{\xi}$ in the direction of the structure vector field, that is $\nabla_{\xi} R_{\xi}=0$. Recently we have some classification theorems for real hypersurfaces in a nonflat complex space form with respect to the parallelism of $R_{\xi}$ (cf. $[3],[4],[5])$.

The main purpose of this paper is to classify real hypersurfaces in a nonflat complex space form $M_{n}(c)$ which satisfies $\nabla_{\xi} R_{\xi}=0$ and at the same time $R_{\xi} \phi S=R_{\xi} S \phi$, where $S$ denotes the Ricci tensor of the hypersurface. Our main result is as follows:

Main Theorem. Let $M$ be a real hypersurface in a complex space form $M_{n}(c)$, $c \neq 0, n \geq 3$ which satisfies $\nabla_{\xi} R_{\xi}=0$. Then $M$ holds $R_{\xi} \phi S=R_{\xi} S \phi$ if and only if $H \xi=0$ or $M$ is locally congruent to one of the following:

(I) in case that $M_{n}(c)=P_{n} \mathbb{C}$,

$\left(A_{1}\right)$ a geodesic hypersphere of radius $r$, where $0<r<\pi / 2$ and $r \neq$ $\pi / 4$,

$\left(A_{2}\right)$ a tube of radius $r$ over a totally geodesic $P_{k} \mathbb{C}$ for some $k \in\{1, \ldots$, $n-2\}$, where $0<r<\pi / 2$ and $r \neq \pi / 4$;

(II) in case that $M_{n}(c)=H_{n} \mathbb{C}$,

$\left(A_{0}\right)$ a horosphere,

$\left(A_{1}\right)$ a geodesic hypersphere or a tube over a complex hyperbolic hyperplane $H_{n-1} \mathbb{C}$,

$\left(A_{2}\right)$ a tube over a totally geodesic $H_{k} \mathbb{C}$ for some $k \in\{1, \ldots, n-2\}$.

We note that every Hopf hypersurface always satisfies condition $R_{\xi} \phi S=$ $R_{\xi} S \phi$

All manifolds in this paper are assumed to be connected and of class $C^{\infty}$ and the real hypersurfaces are supposed to be oriented.

\section{Preliminaries}

We denote by $M_{n}(c), c \neq 0$, be a nonflat complex space form with the Fubini-Study metric $\tilde{g}$ of constant holomorphic sectional curvature $4 c$ and LeviCivita connection $\tilde{\nabla}$. For an immersed $(2 n-1)$-dimensional Riemannian manifold $\tau: M \rightarrow M_{n}(c)$, the Levi-Civita connection $\nabla$ of induced metric and the shape operator $H$ of the immersion are characterized

$$
\tilde{\nabla}_{X} Y=\nabla_{X} Y+g(H X, Y) \nu, \quad \tilde{\nabla}_{X} \nu=-H X
$$


for any vector fields $X$ and $Y$ on $M$, where $g$ denotes the Riemannian metric of $M$ induced from $\tilde{g}$ and $\nu$ a unit normal vector on $M$. In the sequel the indices $i, j, k, l, \ldots$ run over the range $\{1,2, \ldots, 2 n-1\}$ unless otherwise stated. For a local orthonormal frame field $\left\{e_{i}\right\}$ of $M$, we denote the dual 1-forms by $\left\{\theta_{i}\right\}$. Then the connection forms $\theta_{i j}$ are defined by

$$
d \theta_{i}+\sum_{j} \theta_{i j} \wedge \theta_{j}=0, \quad \theta_{i j}+\theta_{j i}=0 .
$$

Then we have

$$
\nabla_{e_{i}} e_{j}=\sum_{k} \theta_{k j}\left(e_{i}\right) e_{k}=\sum_{k} \Gamma_{k i j} e_{k},
$$

where we put $\theta_{i j}=\sum_{k} \Gamma_{i j k} \theta_{k}$. The almost contact metric structure $(\phi=$ $\left.\left(\phi_{i j}\right), \xi=\sum_{i} \xi_{i} e_{i}\right)$ is induced on $M$ by following equation:

$$
J\left(e_{i}\right)=\sum_{j} \phi_{j i} e_{j}+\xi_{i} \nu
$$

The structure tensor $\phi$ and the structure vector $\xi$ satisfy

$$
\begin{aligned}
& \sum_{k} \phi_{i k} \phi_{k j}=\xi_{i} \xi_{j}-\delta_{i j}, \quad \sum_{j} \xi_{j} \phi_{i j}=0, \quad \sum_{i} \xi_{i}^{2}=1, \quad \phi_{i j}+\phi_{j i}=0 \\
& d \phi_{i j}=\sum_{k}\left(\phi_{i k} \theta_{k j}-\phi_{j k} \theta_{k i}-\xi_{i} h_{j k} \theta_{k}+\xi_{j} h_{i k} \theta_{k}\right) \\
& d \xi_{i}=\sum_{j} \xi_{j} \theta_{j i}-\sum_{j, k} \phi_{j i} h_{j k} \theta_{k} .
\end{aligned}
$$

We denote the components of the shape operator or the second fundamental tensor $H$ of $M$ by $h_{i j}$. The components $h_{i j ; k}$ of the covariant derivative of $H$ are given by $\sum_{k} h_{i j ; k} \theta_{k}=d h_{i j}-\sum_{k} h_{i k} \theta_{k j}-\sum_{k} h_{j k} \theta_{k i}$. Then we have the equation of Gauss and Codazzi

(2.2) $\quad R_{i j k l}=c\left(\delta_{i k} \delta_{j l}-\delta_{i l} \delta_{j k}+\phi_{i k} \phi_{j l}-\phi_{i l} \phi_{j k}+2 \phi_{i j} \phi_{k l}\right)+h_{i k} h_{j l}-h_{i l} h_{j k}$,

(2.3) $h_{i j ; k}-h_{i k ; j}=c\left(\xi_{k} \phi_{i j}+\xi_{i} \phi_{k j}-\xi_{j} \phi_{i k}-\xi_{i} \phi_{j k}\right)$,

respectively.

From (2.2) the structure Jacobi operator $R_{\xi}=\left(\Xi_{i j}\right)$ is given by

$$
\Xi_{i j}=\sum_{k, l} h_{i k} h_{j l} \xi_{k} \xi_{l}-\sum_{k, l} h_{i j} h_{k l} \xi_{k} \xi_{l}+c \xi_{i} \xi_{j}-c \delta_{i j} .
$$

From (2.2) the Ricci tensor $S=\left(S_{i j}\right)$ is given by

$$
S_{i j}=(2 n+1) c \delta_{i j}-3 c \xi_{i} \xi_{j}+h h_{i j}-\sum_{k} h_{i k} h_{k j},
$$

where $h=\sum_{i} h_{i i}$.

First we remark: 
Lemma 1 ([5]). Let $U$ be an open set in $M$ and $F$ a smooth function on $U$. We put $d F=\sum_{i} F_{i} \theta_{i}$. Then we have

$$
F_{i j}-F_{j i}=\sum_{k} F_{k} \Gamma_{k i j}-\sum_{k} F_{k} \Gamma_{k j i}
$$

Now we retake a local orthonormal frame field $e_{i}$ in such a way that

- $e_{1}=\xi$,

- $e_{2}$ is in the direction of $\sum_{i=2}^{2 n-1} h_{1 i} e_{i}$,

- $e_{3}=\phi e_{2}$.

Then we have

$$
\xi_{1}=1, \xi_{i}=0(i \geq 2), h_{1 j}=0(j \geq 3) \text { and } \phi_{32}=1 .
$$

We put $\alpha:=h_{11}, \beta:=h_{12}, \gamma:=h_{22}, \varepsilon:=h_{23}$ and $\delta:=h_{33}$.

Hereafter the indices $p, q, r, s, \ldots$ run over the range $\{4,5, \ldots, 2 n-1\}$ unless otherwise stated.

Since $d \xi_{i}=0$, we have

$$
\begin{aligned}
\theta_{12} & =\varepsilon \theta_{2}+\delta \theta_{3}+\sum_{p} h_{3 p} \theta_{p}, \\
\theta_{13} & =-\beta \theta_{1}-\gamma \theta_{2}-\varepsilon \theta_{3}-\sum_{p} h_{2 p} \theta_{p}, \\
\theta_{1 p} & =\sum_{q} \phi_{q p} h_{q 2} \theta_{2}+\sum_{q} \phi_{q p} h_{q 3} \theta_{3}+\sum_{q, r} \phi_{q p} h_{q r} \theta_{r} .
\end{aligned}
$$

We put

$$
\theta_{23}=\sum_{i} X_{i} \theta_{i}, \quad \theta_{2 p}=\sum_{i} Y_{p i} \theta_{i}, \quad \theta_{3 p}=\sum_{i} Z_{p i} \theta_{i}
$$

Then it follows from $d \phi_{2 i}=0$ that $Y_{p i}=-\sum_{q} \phi_{p q} Z_{q i}$ or $Z_{p i}=\sum_{q} \phi_{p q} Y_{q i}$. The equations (2.4) and (2.5) are rewritten as

$$
\begin{aligned}
& \Xi_{i j}=-\alpha h_{i j}+h_{1 i} h_{1 j}+c \delta_{i 1} \delta_{j 1}-c \delta_{i j}, \\
& S_{i j}=h h_{i j}-\sum_{k} h_{i k} h_{j k}-3 c \delta_{i 1} \delta_{j 1}+(2 n+1) c \delta_{i j},
\end{aligned}
$$

respectively.

\section{Real hypersurfaces satisfying $\nabla_{\xi} R_{\xi}=0$ and $R_{\xi} \phi S=R_{\xi} S \phi$}

First we assume that $\nabla_{\xi} R_{\xi}=0$. The components $\Xi_{i j ; k}$ of the covariant derivativation of $R_{\xi}=\left(\Xi_{i j}\right)$ is given by

$$
\sum_{k} \Xi_{i j ; k} \theta_{k}=d \Xi_{i j}-\sum_{k} \Xi_{k j} \theta_{k i}-\sum_{k} \Xi_{i k} \theta_{k j}
$$


Substituting (2.9) into the above equation we have

$$
\begin{aligned}
\sum_{k} \Xi_{i j ; k} \theta_{k}= & -(d \alpha) h_{i j}-\alpha d h_{i j}+\left(d h_{1 i}\right) h_{1 j}+h_{1 i}\left(d h_{1 j}\right) \\
& +\alpha \sum_{k} h_{k j} \theta_{k i}-\alpha h_{1 j} \theta_{1 i}-\beta h_{1 j} \theta_{2 i}-c \delta_{j 1} \theta_{1 i} \\
& +\alpha \sum_{k} h_{i k} \theta_{k j}-\alpha h_{1 i} \theta_{1 j}-\beta h_{1 i} \theta_{2 j}-c \delta_{i 1} \theta_{1 j} .
\end{aligned}
$$

In the following, we assume that $\beta \neq 0$.

Our assumption $\nabla_{\xi} R_{\xi}=0$ is equivalent to $\Xi_{i j ; 1}=0$, which can be stated as follows:

$$
\begin{aligned}
& \varepsilon=0, \quad \alpha \delta+c=0, \quad h_{3 p}=0 \\
& \left(\beta^{2}-\alpha \gamma\right)_{1}-2 \alpha \sum_{p} h_{2 p} Y_{p 1}=0 \\
& \left(\beta^{2}-\alpha \gamma-c\right) X_{1}+\alpha \sum_{p} h_{2 p} Z_{p 1}=0 \\
& \left(\alpha h_{2 p}\right)_{1}+\alpha \sum_{q} h_{p q} Y_{q 1}+\left(\beta^{2}-\alpha \gamma\right) Y_{p 1}-\alpha \sum_{q} h_{2 q} \Gamma_{q p 1}=0 \\
& \alpha h_{2 p} X_{1}-\sum_{q}\left(\alpha h_{q p}+c \delta_{p q}\right) Z_{q 1}=0 \\
& \left(\alpha h_{p q}\right)_{1}-\alpha h_{2 q} Y_{p 1}-\alpha \sum_{r} h_{r q} \Gamma_{r p 1}-\alpha h_{2 p} Y_{q 1}-\alpha \sum_{r} h_{p r} \Gamma_{r q 1}=0 .
\end{aligned}
$$

Hereafter we shall use (3.2) without quoting.

Furthermore we assume that $R_{\xi} \phi S=R_{\xi} S \phi$. Under the assumption $\nabla_{\xi} R_{\xi}=$ 0 , we have the following additional equations

$$
\begin{aligned}
& \beta^{2}-\alpha \gamma-c=0, \\
& \tilde{R}_{\xi} \tilde{\phi} A=0, \\
& \tilde{R}_{\xi} \tilde{\phi} \tilde{S}=\tilde{R}_{\xi} \tilde{S} \tilde{\phi},
\end{aligned}
$$

where $A={ }^{t}\left(h_{24}, h_{25}, \ldots, h_{2,2 n-1}\right), \tilde{R}_{\xi}=\left(\Xi_{p q}\right), \tilde{\phi}=\left(\phi_{p q}\right), \tilde{S}=\left(S_{p q}\right)$. From (3.2) and (3.8) we note

$$
\delta \neq \gamma
$$

Now, properly speaking, we denote the equation (2.3) by ( $i j k)$ simply. Then we have the following equations (112)-(q3p).

$$
\begin{aligned}
& \alpha_{2}-\beta_{1}=0, \\
& \beta_{2}-\gamma_{1}-2 \sum_{p} h_{2 p} Y_{p 1}=0,
\end{aligned}
$$




$$
(\alpha-\delta) \gamma-\beta X_{2}+(\gamma-\delta) X_{1}-\beta^{2}-\sum_{p} h_{2 p} Z_{p 1}=-c,
$$

$$
\alpha_{3}+3 \beta \delta-\alpha \beta+\beta X_{1}=0
$$$$
\beta_{3}+\gamma \delta+(\gamma-\delta) X_{1}-\beta^{2}-\sum_{p} h_{2 p} Z_{p 1}=0
$$

$\beta X_{3}+\delta_{1}=0$

$\gamma_{3}-2 \beta \delta+2 \sum_{p} h_{2 p} Y_{p 3}+(\gamma-\delta) X_{2}-\beta \gamma-\sum_{p} h_{2 p} Z_{p 2}=0$

$\sum_{p} h_{2 p} Z_{p 3}-\delta_{2}-(\gamma-\delta) X_{3}=0$

$\alpha_{p}+\beta Y_{p 1}=0$

$\beta_{p}+2 \sum_{q, r} h_{2 q} \phi_{r q} h_{r p}+\beta Y_{p 2}+\alpha \sum_{q} \phi_{q p} h_{2 q}=0$

$2 \delta h_{2 p}-\beta Y_{p 3}-\alpha h_{2 p}+\beta X_{p}=0$,

$\gamma_{p}+2 \sum_{q} h_{2 q} Y_{q p}-h_{2 p 2}-\sum_{q} h_{q p} Y_{q 2}+\beta \sum_{q} \phi_{q p} h_{2 q}+\gamma Y_{p 2}$

$$
+\sum_{q} h_{2 q} \Gamma_{q p 2}=0
$$

$$
\begin{aligned}
& \delta X_{p}+\beta h_{2 p}-\gamma X_{p}+\sum_{q} h_{2 q} Z_{q p}-h_{2 p 3}-\sum_{q} h_{q p} Y_{q 3} \\
& \quad+\gamma Y_{p 3}+\sum_{q} h_{2 q} \Gamma_{q p 3}=0
\end{aligned}
$$

$$
\delta_{p}+h_{2 p} X_{3}-\sum_{q} h_{q p} Z_{q 3}+\delta Z_{p 3}=0
$$

$$
\beta_{p}+\sum_{q, r} h_{2 q} \phi_{r q} h_{r p}-h_{2 p 1}-\sum_{q} h_{q p} Y_{q 1}+\gamma Y_{p 1}+\sum_{q} h_{2 q} \Gamma_{q p 1}=0
$$

$$
\delta h_{2 p}-\alpha h_{2 p}+\beta X_{p}-h_{2 p} X_{1}+\sum_{q} h_{q p} Z_{q 1}-\delta Z_{p 1}=0,
$$

$(1 p q)$

$$
\delta X_{p}+\beta h_{2 p}-\gamma X_{p}+\sum_{q} h_{2 q} Z_{q p}+h_{2 p} X_{2}-\sum_{q} h_{p q} Z_{q 2}+\delta Z_{p 2}=0,
$$

$$
2 \sum_{r, s} h_{r p} \phi_{s r} h_{s q}-\alpha \sum_{r} \phi_{r p} h_{r q}+\alpha \sum_{r} \phi_{r q} h_{r p}
$$

$$
-\beta Y_{p q}+\beta Y_{q p}=-2 c \phi_{p q},
$$

$(2 p q)$

$$
h_{2 p q}+\sum_{r} h_{r p} Y_{r q}-\beta \sum_{r} \phi_{r p} h_{r q}-\gamma Y_{p q}-\sum_{r} h_{2 r} \Gamma_{r p q}-h_{2 q p}
$$




$$
-\sum_{r} h_{r q} Y_{r p}+\beta \sum_{r} \phi_{r q} h_{r p}+\gamma Y_{q p}+\sum_{r} h_{2 r} \Gamma_{r q p}=0,
$$

$(q 1 p)$

$$
\begin{aligned}
& \sum_{r, s} h_{r q} \phi_{s r} h_{s p}-\alpha \sum_{r} \phi_{r q} h_{r p}-\beta Y_{q p}-h_{p q 1} \\
& \quad+h_{2 q} Y_{p 1}+\sum_{r} h_{r q} \Gamma_{r p 1}+h_{2 p} Y_{q 1}+\sum_{r} h_{r p} \Gamma_{r q 1}=c \phi_{p q}
\end{aligned}
$$

$(q 3 p)$

$$
\begin{aligned}
& h_{3 q p}-\varepsilon Y_{q p}-\delta Z_{q p}-\sum_{r} h_{3 r} \Gamma_{r q p}-h_{2 q} X_{p}+\sum_{r} h_{q r} Z_{r p}-h_{q p 3} \\
& \quad+h_{q 2} Y_{p 3}+h_{q 3} Z_{p 3}+\sum_{r} h_{q r} \Gamma_{r p 3}+h_{p 2} Y_{q 3}+h_{p 3} Z_{q 3} \\
& \quad+\sum_{r} h_{p r} \Gamma_{r q 3}=0 .
\end{aligned}
$$

Remark 1. We did not write $(p 2 q),(3 p q)$ and $(p q r)$ since we need not use them.

\section{Formulas and lemmas}

In this section we study the crucial case where $\beta \neq 0$. By (3.6) and (31p) we have

$$
\beta X_{p}=(\alpha-\delta) h_{2 p}
$$

This and (13p) imply that

$$
\beta Y_{p 3}=\delta h_{2 p}
$$

The equation (3.9) can be rewritten as

$$
\sum_{q, r}\left(\alpha h_{p q}+c \delta_{p q}\right) \phi_{q r} h_{r 2}=0
$$

which, together with (4.2), implies

$$
\beta \sum_{q}\left(h_{p q}-\delta \delta_{p q}\right) Z_{q 3}=\delta \sum_{q, r}\left(h_{p q}-\delta \delta_{p q}\right) \phi_{q r} Y_{r 3}=0 .
$$

Hence it follows from (33p) and (1p1) that

$$
\delta_{p}=-h_{2 p} X_{3} \text { and } \alpha_{p}=-\beta Y_{p 1} .
$$

Thus since (4.4) and $\alpha_{p} \delta+\alpha \delta_{p}=0$ obtained from (3.2) we have

$$
\beta \delta Y_{p 1}=-\alpha h_{2 p} X_{3},
$$

and so $\sum_{p} h_{2 p} Z_{p 1}=0$. By (4.2), we have

$$
\sum_{p} h_{2 p} Z_{p 3}=\sum_{p, q} h_{2 p} \phi_{p q} Y_{q 3}=\frac{\delta}{\beta} \sum_{p, q} h_{2 p} \phi_{p q} h_{2 q}=0 .
$$


From (3.6), (4.3) and (4.5) we have

$$
h_{2 p} X_{1}=0 \text {. }
$$

Now we shall prove the following key lemma.

Lemma 2. $H\left(e_{2}\right) \in \operatorname{span}\left\{e_{1}, e_{2}\right\}$.

Proof. Suppose that $h_{2 p} \neq 0$. Then from (4.7) we have $X_{1}=0$. We can select the vector $e_{4}$ so that $h_{24} \neq 0$ and $h_{25}=\cdots=h_{2,2 n-1}=0$. We put $e_{5}:=\phi e_{4}$ and $\rho:=h_{24}(\neq 0)$. Note that $\phi_{54}=1$. Then by (4.3) we have

$$
h_{55}=\delta, \quad h_{p 5}=0(p \neq 5) .
$$

Put $p=5$ in $(32 p)$. Then since above equation and (4.1) we have $X_{5}=0$ and so $Z_{45}=0$. Thus we have $Y_{55}=0$. Furthermore, put $p=q=5$ in $(q 1 p)$. Then, since $\Gamma_{551}=Y_{55}=0$, we have

$$
\alpha_{1}=\delta_{1}=0 .
$$

Thus, from (313), (323), (4.6) and (112) we have

$$
\begin{aligned}
& X_{3}=0, \\
& \alpha_{2}=\delta_{2}=0, \\
& \beta_{1}=0 .
\end{aligned}
$$

By (4.4) and (4.9) we have $\alpha_{p}=\delta_{p}=0$. Thus it follows from (1p1) that

$$
\alpha_{p}=\delta_{p}=Y_{p 1}=Z_{p 1}=0 .
$$

Now we put $F=\alpha, i=1$ and $j=p$ in Lemma 1. Then, from (2.7), (4.8), (4.10) and (4.12) we have

$$
0=\alpha_{1 p}-\alpha_{p 1}=\sum_{k} \alpha_{k} \Gamma_{k 1 p}-\sum_{k} \alpha_{k} \Gamma_{k p 1}=\alpha_{3}\left(\Gamma_{31 p}-\Gamma_{3 p 1}\right)=\alpha_{3} h_{2 p} .
$$

Thus we have $\alpha_{3}=0$. Hence it follows from (4.8), (4.10) and (4.12) that $\alpha$ and $\delta$ are constants, which, together with (113), imply

$$
\alpha=3 \delta \text {. }
$$

On the other hand, taking account of the coefficient of $\theta_{1} \wedge \theta_{3}$ in the exterior derivative of $\theta_{23}$, we have $X_{2}=-2 \beta$. Thus, from (312) and (4.13) we have

$$
2 \delta \gamma+\beta^{2}=-c \text {. }
$$

Hence it follows from (3.8) that $\beta$ and $\gamma$ are constants. From (3.5) and (4.12) we have

$$
h_{2 p 1}-\sum_{q} h_{2 q} \Gamma_{q p 1}=0 .
$$

This, together with $(21 p)$, implies $\rho \delta=0$, which is a contradiction.

Remark 2. Lemma 2 has been already proved in [5]. However in the case of our condition by using the equation (3.8) we have a short proof. 
Owing to Lemma 2 the matrix $\left(h_{p q}\right)$ is diagonalizable, that is, for a suitable choice of a orthonormal frame field $\left\{e_{p}\right\}$ we can set

$$
h_{p q}=\lambda_{p} \delta_{p q} .
$$

Then it is easy to see

$$
\begin{aligned}
& \tilde{R}_{\xi}=-\left(\left(\alpha \lambda_{p}+c\right) \delta_{p q}\right), \\
& \tilde{S}=\left(\left(h \lambda_{p}-\left(\lambda_{p}\right)^{2}+(2 n+1) c\right) \delta_{p q}\right) .
\end{aligned}
$$

Here we shall sum up all equations obtained from Lemma 2.

From (4.1), (4.2) and (4.4) we have

$$
X_{p}=Y_{p 1}=Z_{p 1}=Y_{p 3}=Z_{p 3}=0, \quad \alpha_{p}=\delta_{p}=0 .
$$

Put $p=q$ in (3.7). Then we have

$$
\left(\alpha \lambda_{p}\right)_{1}=0 .
$$

Moreover, from (112)-(32p) we have

$$
\begin{aligned}
& \alpha_{2}-\beta_{1}=0 \\
& \beta_{2}-\gamma_{1}=0 \\
& \gamma \delta+\beta X_{2}-(\gamma-\delta) X_{1}=0 \\
& \alpha_{3}+3 \beta \delta-\alpha \beta+\beta X_{1}=0 \\
& \beta_{3}-\alpha \delta+\gamma \delta+(\gamma-\delta) X_{1}-\beta^{2}=c \\
& \delta_{1}+\beta X_{3}=0 \\
& \gamma_{3}-2 \beta \delta+(\gamma-\delta) X_{2}-\beta \gamma=0 \\
& \delta_{2}+(\gamma-\delta) X_{3}=0 \\
& \beta_{p}=0 \\
& Y_{p 2}=0 \\
& \gamma_{p}=0
\end{aligned}
$$

It follows from $(q 1 p)$ and (3.7) that

$$
\alpha \beta Y_{q p}=\alpha \lambda_{p} \lambda_{q} \phi_{p q}-\alpha^{2} \lambda_{p} \phi_{p q}+\alpha_{1} \lambda_{p} \delta_{p q}-c \alpha \phi_{p q} .
$$

From this, $(2 p q)$ and $(q 3 p)$ we have

$$
\begin{aligned}
\beta^{2}\left(\lambda_{p}+\lambda_{q}\right) \phi_{p q} & -\left(\lambda_{p}-\gamma\right)\left(\lambda_{p} \lambda_{q}-\alpha \lambda_{q}-c\right) \phi_{p q} \\
& -\left(\lambda_{q}-\gamma\right)\left(\lambda_{p} \lambda_{q}-\alpha \lambda_{p}-c\right) \phi_{p q}=0,
\end{aligned}
$$

(4.30) $\left(\lambda_{q}-\delta\right)\left(\alpha\left(\left(\lambda_{q}\right)^{2}-\alpha \lambda_{q}-c\right) \delta_{p q}+\alpha_{1} \lambda_{q} \phi_{p q}\right)-\alpha \beta\left(h_{q p 3}+\left(\lambda_{p}-\lambda_{q}\right) \Gamma_{q p 3}\right)=0$.

If $p=q$ in above equation, then we have

$$
\left(\lambda_{p}-\delta\right)\left(\left(\lambda_{p}\right)^{2}-\alpha \lambda_{p}-c\right)-\beta\left(\lambda_{p}\right)_{3}=0 .
$$




\section{Proof of Main Theorem}

In this section we prove Main Theorem.

We first prove $\beta=0$. Suppose that $\beta \neq 0$. It follows from (4.22) that (3.10) is equivalent to

$$
\rho_{p}\left(\sigma_{q}-\sigma_{p}\right) \phi_{p q}=0
$$

where $\rho_{p}=\alpha \lambda_{p}+c, \sigma_{p}=h \lambda_{p}-\left(\lambda_{p}\right)^{2}+(2 n+1) c$. Therefore if $\phi_{q p} \neq 0$, then we have

$$
\left(\lambda_{p}-\lambda_{q}\right)\left(\alpha \lambda_{p}+c\right)\left(h-\lambda_{q}-\lambda_{p}\right)=0 .
$$

Here we assert that if $\phi_{p q} \neq 0$, then $\lambda_{p}=\lambda_{q}$. To prove this, we assume that there exist indices $p$ and $q$ such that

$$
\phi_{p q} \neq 0, \quad \lambda_{p}-\lambda_{q} \neq 0 .
$$

First we prepare three lemmas.

Lemma 3. $\alpha \alpha_{1}-(\alpha \gamma)_{1}=0$.

Proof. From (5.1) we have

$$
\begin{aligned}
& \left(\alpha \lambda_{p}+c\right)\left(h-\lambda_{p}-\lambda_{q}\right)=0, \\
& \left(\alpha \lambda_{q}+c\right)\left(h-\lambda_{p}-\lambda_{q}\right)=0 .
\end{aligned}
$$

Thus if $h-\lambda_{p}-\lambda_{q} \neq 0$, then we have $\lambda_{p}=\lambda_{q}=0$, which is a contradiction. Hence we have $\alpha h-\alpha \lambda_{p}-\alpha \lambda_{q}=0$. Taking account of the coefficient of $\theta_{1}$ in the exterior derivative of this, it follows from (4.16) that

$$
(\alpha h)_{1}=0 \text {. }
$$

From (4.26) we have $\left(\alpha \sum_{p} \lambda_{p}\right)_{1}=0$. Combining this equation with $h=$ $\alpha+\gamma+\delta+\sum_{p} \lambda_{p}$, we have

$$
(\alpha(h-\alpha-\gamma-\delta))_{1}=0 .
$$

Eliminating $h$ from this Lemma 3 follows from (5.2).

Lemma 4. $\alpha_{1} \neq 0$ and $\alpha+\gamma-\delta=0$.

Proof. From (4.24) we have $2 \beta \beta_{1}-(\alpha \gamma)_{1}=0$. Hence it follows from Lemma 4 that

$$
\alpha \alpha_{1}+\beta \beta_{1}=0 .
$$

On the other hand, by (4.32) and (4.34) we have $(\gamma-\delta) \delta_{1}-\beta \delta_{2}=0$, and therefore $(\gamma-\delta) \alpha_{1}-\beta \alpha_{2}=0$. Thus we have

$$
(\alpha+\gamma-\delta) \alpha_{1}=0 \quad \text { and } \quad(\alpha+\gamma-\delta) \beta_{1}=0 .
$$

Suppose that

$$
\alpha_{1}=0
$$

It follows from (3.8) that

$$
\left(\beta^{2}-\alpha \gamma-c\right)_{3}=2 \beta \beta_{3}-\gamma \alpha_{3}-\alpha \gamma_{3}=0 .
$$


From (4.19)-(4.21), (4.23) and (3.8) we have the following:

$$
\begin{aligned}
& \delta \gamma+\beta X_{2}-(\gamma-\delta) X_{1}=0, \\
& \alpha_{3}+3 \beta \delta-\alpha \beta+\beta X_{1}=0, \\
& \beta_{3}+(\gamma-\delta) X_{1}+\gamma \delta-\alpha \gamma-c=0, \\
& \gamma_{3}-2 \beta \delta+(\gamma-\delta) X_{2}+\beta \gamma=0 .
\end{aligned}
$$

Substituting of (5.8)-(5.10) into (5.6) we have

$$
X_{1}=4 \alpha,
$$

by virtue of (3.11) and (5.7). Substituting of this equation into (5.7)-(5.9) we have

$$
\begin{aligned}
& \beta X_{2}=4 \alpha(\gamma-\delta)-\delta \gamma, \\
& \alpha_{3}+3 \beta \delta+3 \alpha \beta=0, \\
& \beta_{3}+3 \alpha \gamma-3 \alpha \delta+\gamma \delta=0 .
\end{aligned}
$$

It follows from $(4.23),(3.8)$ and $(5.12)$ that

$$
\alpha \gamma_{3}+\beta(3 \alpha \gamma-6 \alpha \delta-\gamma \delta)=0
$$

From (4.22), (5.3) and (5.5) we have $X_{3}=0$ and $\beta_{1}=0$ and therefore $\alpha_{2}=$ $\delta_{2}=0$ because of (4.17). Hence, by (3.8) and (5.5), we have $\gamma_{1}=0$, and so $\beta_{2}=0$.

Now put $F=\alpha$ and $\beta$ in Lemma 1. Then we have

$$
\alpha_{3}\left(\gamma+X_{1}\right)=0, \quad \beta_{3}\left(\gamma+X_{1}\right)=0 .
$$

If $\gamma+X_{1} \neq 0$, then we have $\alpha_{3}=\beta_{3}=0$. It follows from (4.15) and (4.25) that $\alpha, \beta$ and $\delta$ are constants and that $\alpha_{i}=\beta_{i}=0$ for $i=1,2$. Furthermore, by (3.8) we see that $\gamma$ is a constant. Thus from (5.13)-(5.15) we have

$$
\begin{aligned}
& \alpha+\delta=0, \\
& 3 \alpha \gamma-3 \alpha \delta+\gamma \delta=0, \\
& 3 \alpha \gamma-6 \alpha \delta-\gamma \delta=0
\end{aligned}
$$

Hence, by (3.2) and (3.8) we have $\alpha^{2}-c=0$ and $2 \beta^{2}+c=0$, which is a contradiction. Therefore $X_{1}=-\gamma$, which, together with (5.11), implies $\gamma=-X_{1}=-4 \alpha$. Thus it follows from (5.13) that $\gamma_{3}=-4 \alpha_{3}=12 \beta(\delta+\alpha)$. Therefore from (5.15) we have a contradiction $\alpha \delta=0$.

Lemma 5. $X_{1}=0$.

Proof. Suppose that $X_{1} \neq 0$. Then from (4.29) we have

$$
\left(-\lambda_{p} \lambda_{q}+2 c\right)\left(\lambda_{p}+\lambda_{q}\right)+2(\alpha+\gamma) \lambda_{p} \lambda_{q}-2 c \gamma=0 .
$$

Multiply above equation by $\alpha^{3}$ and take account of the coefficient of $\theta_{1}$ in the exterior derivative of this equation. Then, from Lemma 3 and (4.16) we have

$$
\alpha \lambda_{p}+\alpha \lambda_{q}-\alpha \gamma+\alpha^{2}=0 \text {. }
$$


Again, taking account of the coefficient of $\theta_{1}$ in the exterior derivative of above equation, we have $\alpha \alpha_{1}=0$, which is a contradiction.

Consequently, for all $p, q$ such that $\phi_{p q} \neq 0$, we have $\lambda_{p}=\lambda_{q}$. We take $p, q$ such that $\phi_{p q} \neq 0$. Then by (4.29) we have

$$
\beta^{2} \lambda_{p}-\left(\lambda_{p}-\gamma\right)\left(\left(\lambda_{p}\right)^{2}-\alpha \lambda_{p}-c\right)=0
$$

Furthermore, from $(q 3 p),(4.28)$ and $(4.16)$ we have

$$
\left(\lambda_{p}\right)_{1}\left(\lambda_{p}-\delta\right)=0 .
$$

It follows from (4.16) and Lemma 4 that $\left(\lambda_{p}\right)_{1} \neq 0$. Hence we have $\lambda_{p}=\delta$, which, together with (3.8), (3.11) and (5.16), implies $\alpha+\delta=0$. Therefore $\alpha$ and $\delta$ are constants, which is a contradiction.

Taking account of the coefficient of $\theta_{3}$ in the exterior derivative of equation $\alpha+\gamma-\delta=0$ and making use of (4.19), (4.20), (4.23) and Lemma 5, we have

$$
\alpha(\gamma-\delta)(\gamma(\alpha-\delta))+\beta^{2}\left(\alpha^{2}+\alpha \delta-3 \delta^{2}\right)=0
$$

Again taking account of the coefficient of $\theta_{1}$ in the exterior derivative of (5.17) and taking account of (3.2) and (5.3), we have

$$
\alpha^{4}+4 \alpha^{2} c-2 \delta^{2} c+3 c^{2}+\beta^{2}\left(\alpha^{2}+3 \delta^{2}\right)=0 .
$$

Eliminating $\beta$ from (5.17) and (5.18), we have a polynomial $4 \alpha^{4}+7 c \alpha^{2}+4 c=0$ of degree four with respect to $\alpha$. This shows that $\alpha$ is a constant, which contradicts Lemma 4 . Consequently we proved $\beta=0$.

Since (2.6) and $\beta=0$, we see that $\alpha$ is a constant in $M$ (see [6]). Thus from (3.1) our assumption $\Xi_{i j ; 1}=0$ is equivalent to $\alpha h_{i j ; 1}=0$. Put $j=1$ in (2.3). Then by above equation we have $\alpha h_{i 1 ; k}=-c \alpha \phi_{i k}$. Therefore since (2.1) and $d \xi_{i}=0$, we have

$$
\alpha \sum_{k, l} h_{i k} \phi_{l k} h_{k j}+\alpha^{2} \sum_{k} \phi_{k i} h_{k j}=-\alpha h_{i 1 ; j}=c \alpha \phi_{i j}
$$

which implies that $\alpha^{2}(\phi H-H \phi)=0$. Owing to Okumura's work or Montiel and Romero's work stated in Introduction, we complete the proof of our Main Theorem.

Remark 3. In previous papers [4] and [5], we classified real hypersurfaces which satisfy $\nabla_{\xi} R_{\xi}=0$ and some several conditions in $M_{n}(c), c \neq 0, n \geq 3$. Combining these results we have the following:

Let $M$ be a real hypersurface in a complex space form $M_{n}(c), c \neq 0, n \geq 3$ which satisfies $\nabla_{\xi} R_{\xi}=0$. Then the following conditions are mutually equivalent.

(i) $M$ is locally congruent to type $A$.

(ii) $R_{\xi} S=S R_{\xi}$.

(iii) $R_{\xi} \phi S=S \phi R_{\xi}$.

(iv) $R_{\xi} \phi S=R_{\xi} S \phi$. 
Remark 4. Recently the second author extended Main Theorem to the case $n=2[8]$.

\section{References}

[1] J. Berndt, Real hypersurfaces with constant principal curvatures in complex hyperbolic spaces, J. Reine Angew. Math. 395 (1989), 132-141.

[2] T. E. Cecil and P. J. Ryan, Focal sets and real hypersurfaces in complex projective space, Trans. Amer. Math. Soc. 269 (1982), no. 2, 481-499.

[3] U.-H. Ki and H. Kurihara, Real hypersurfaces with cyclic-parallel structure Jacobi operators in a nonflat complex space form, Bull. Aust. Math. Soc. 81 (2010), no. 2, 260-273.

[4] U.-H. Ki, H. Kurihara, S. Nagai and R. Takagi, Characterizations of real hypersurfaces of type $A$ in a complex space form in terms of the structure Jacobi operator, Toyama Math. J. 32 (2009), 5-23.

[5] U.-H. Ki, H. Kurihara, and R. Takagi, Jacobi operators along the structure flow on real hypersurfaces in a nonflat complex space form, Tsukuba J. Math. 33 (2009), no. 1, $39-56$.

[6] U.-H. Ki and Y. J. Suh, On real hypersurfaces of a complex space form, Math. J. Okayama Univ. 32 (1990), 207-221.

[7] M. Kimura, Real hypersurfaces and complex submanifolds in complex projective space, Trans. Amer. Math. Soc. 296 (1986), no. 1, 137-149.

[8] H. Kurihara, The structure Jacobi operator for real hypersurfaces in the complex projective plane and the complex hyperbolic plane, Tsukuba J. Math. 35 (2011), 53-66.

[9] M. Montiel and A. Romero, On some real hypersurfaces of a complex hyperbolic space, Geom. Dedicata 20 (1986), no. 2, 245-261.

[10] M. Okumura, On some real hypersurfaces of a complex projective space, Trans. Amer. Math. Soc. 212 (1975), 355-364.

[11] M. Ortega, J. D. Pérez, and F. G. Santos, Non-existence of real hypersurfaces with parallel structure Jacobi operator in nonflat complex space forms, Rocky Mountain J. Math. 36 (2006), no. 5, 1603-1613.

[12] J. D. Pérez, F. G. Santos, and Y. J. Suh, Real hypersurfaces in complex projective spaces whose structure Jacobi operator is D-parallel, Bull. Belg. Math. Soc. Simon Stevin 13 (2006), no. 3, 459-469.

[13] R. Takagi, On homogeneous real hypersurfaces in a complex projective space, Osaka J. Math. 10 (1973), 495-506.

[14] _ Real hypersurfaces in a complex projective space with constant principal curvatures I, II, J. Math. Soc. Japan 27 (1975), 43-53, 507-516.

U-HANG KI

DePARTMENT OF MATHEMATiCS

KYUNGPOOK NATIONAL University

DAEgu 702-701, Korea

E-mail address: uhangki2005@yahoo.co.kr

Hiroyuki Kurihara

Department of Liberal Arts and Engineering Sciences

Hachinohe National College of Technology

HaChinOHe, AOMORI 039-1192, JAPAN

E-mail address: kurihara-g@hachinohe-ct.ac.jp 